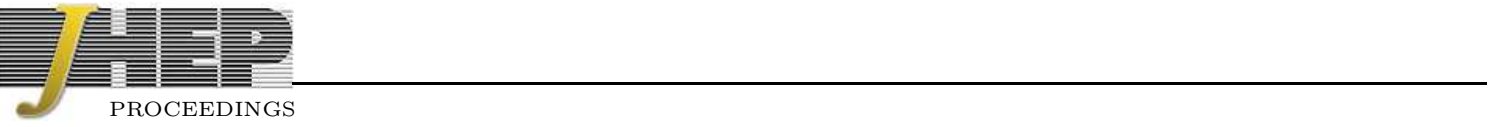

\title{
Cosmological billiards and oxidation
}

\author{
S. de Buyl ${ }^{a *}$, M. Henneaux ${ }^{a, b}$, B. Julia ${ }^{c}$ and Louis Paulot ${ }^{a}$ \\ a Physique Théorique et Mathématique, Université Libre de Bruxelles, \\ C.P. 231; B-1050, Bruxelles, Belgium \\ ${ }^{b}$ Centro de Estudios Científicos, \\ Casilla 1469, Valdivia, Chile \\ c Laboratoire de Physique Théorique de l'Ecole Normale Supérieure, \\ 24, rue Lhomond, F-75231 Paris CEDEX 05, France \\ E-mail: sdebuyl@ulb.ac.be, henneaux@ulb.ac.be, Bernard.Julia@lpt.ens.fr, \\ Louis.Paulot@ulb.ac.be
}

\begin{abstract}
We show how the properties of the cosmological billiards provide useful information (spacetime dimension and $p$-form spectrum) on the oxidation endpoint of the oxidation sequence of gravitational theories. We compare this approach to the other available methods: $G L(n, R)$ subgroups and the superalgebras of dualities.
\end{abstract}

\section{Introduction}

It has been shown recently that the dynamics of gravitational theories can be described, in the vicinity of a spacelike singularity, as a billiard motion in a region of hyperbolic space bounded by hyperplanes [1]-[6]. For gravitational theories that reduce upon toroidal compactification to $D=3$ dimensions to 3 -dimensional gravity coupled to a symmetric space non-linear $\sigma$-model, the billiard turns out to have remarkable regularity properties: it is the fundamental Weyl chamber of the overextension [7, 8] of the symmetry group $\mathcal{U}_{3}$ [9]-11] that emerges in 3 dimensions or, when the real form $\mathcal{U}_{3}$ is not split, of its maximal split subalgebra 12.

An interesting feature of the billiard region is that it is invariant under toroidal dimensional reduction to any dimension $D \geq 3$ [11]. Knowing the billiard region in $D=3$ dimensions can then be used as a tool to determine the possible higher dimensional parents of the theory. This problem of going up in dimension is known as the oxidation or disintegration problem and goes back to the early days of supergravity and has been studied repeatedly $[7$, 13, 14]. It has been thoroughly investigated recently by means of group theory techniques [15]-[17]. We show here that the billiard approach gives direct information

\footnotetext{
*Aspirant du Fonds National de la Recherche Scientifique, Belgique
} 
on possible obstructions to oxidation and, when oxidation is possible, restricts efficiently the maximal dimension(s) of oxidation as well as determines the full $p$-form spectrum of the maximally oxidized theory. We consider both split and non-split $\mathcal{U}_{3}$-groups. For non maximally non-compact groups (ie non-split), this approach is complementary to the general method of general linear subgroups of [14] known as the "A-chain" method. We also point out that the same information can be extracted from the superalgebra approach to the problem [18]-20].

\section{Walls associated with simple roots and oxidation}

The walls bounding the billiards have different origins [9, 6]: they can be symmetry or curvature walls, related to the Einstein-Hilbert action; or they can be $p$-form walls (electric or magnetic), related to the $p$-form part of the action. The key to the derivation of the oxidation constraints is to investigate how the billiard walls behave upon dimensional reduction. Although the billiard region is invariant, the formal origin of the walls may change. E.g., a symmetry wall in $D$ dimensions may appear as a 1-form wall associated with the Kaluza-Klein graviphoton(s) in lower dimensions [9].

The translation rules relating walls in higher dimensions to walls in lower dimensions have been worked out in [11, 12]. To recall them, we denote by $\bar{B}$ the restricted root system of the real form $\mathcal{U}_{3}$. This restricted root system may be reduced or non reduced, in the latter case it is of $B C_{r}$-type [21]. The billiard region is the fundamental Weyl chamber of the overextension $\bar{B}^{\wedge \wedge}$ [12] obtained by adding to the simple roots of the finite root system $\bar{B}$, the affine root $\alpha_{0}$ and the "overextended root" $\alpha_{-1}$, which is attached to the affine root with a single link. There are at most two different root lengths, except when the underlying finite root system is non reduced, in which case one has three different root lengths. The highest root is always a long root ("very long" root in the $B C_{r}$-case) [12].

From the point of view of the three-dimensional action (Einstein-Hilbert action $+\mathcal{U}_{3}$ non linear $\sigma$-model action), these roots have the following interpretation ${ }^{1}$ : (i) the overextended root is the symmetry wall $\beta^{2}-\beta^{1}$ and involves only gravitational variables; (ii) the affine root corresponds to the dominant magnetic wall $\beta^{1}-\theta(\phi)$ where $\theta(\phi)$ is the highest root of $\bar{B}$; it involves both the dilatons and the gravitational variable $\beta^{1}$; and (iii) the simple roots of $\bar{B}$ correspond to the dominant electric walls defined by the 0 -forms (axions) and involve the dilatons only.

In $D=d+1>3$ dimensions, these walls appear as follows. (i) The overextended root remains a symmetry wall ${ }^{2}$, namely $\beta^{d}-\beta^{d-1}$; (ii) The affine root becomes the symmetry wall $\beta^{d-1}-\beta^{d-2}$. (iii) The (long) roots of $\bar{B}$ connected to the affine root by a chain of single links form a chain of symmetry walls $\beta^{d-2}-\beta^{d-3}, \cdots, \beta^{2}-\beta^{1}$. The other roots correspond to $p$-form walls of the form $\beta^{1}+\cdots+\beta^{p}+\frac{1}{2} \sum_{\alpha} \lambda_{\alpha}^{(p)} \phi^{\alpha}$ (electric walls) or

\footnotetext{
${ }^{1}$ As in our previous work, we denote the logarithmic scale factors of the spatial metric by $\beta^{1}$ and $\beta^{2}$. The scalar fields of the $\sigma$-model Iwasawa-split into the dilatons $\phi^{\Delta}(\Delta=1, \cdots, r$ where $r$ is the real rank) and the axions (0-forms) $\chi^{A}$.

${ }^{2}$ Scale factors get redefined as one changes the spacetime dimension but we shall keep the same notation since the context is in each case clear.
} 
$\beta^{1}+\cdots+\beta^{d-p-1}-\frac{1}{2} \sum_{\alpha} \lambda_{\alpha}^{(p)} \phi^{\alpha}$ (magnetic walls) ${ }^{3}$. In these equations, the $\lambda_{\alpha}^{(p)}$ are the couplings to the dilatons $\phi^{\alpha}$ present in $D$ dimensions (if any).

\section{Obstructions to oxidation}

A crucial feature of the gravitational walls (symmetry and curvature walls) is that they are non degenerate. By contrast, the $p$-form walls may come with a non trivial multiplicity since one can include many $p$-forms with the same degree and same dilaton couplings.

It follows from this observation that a "necessary condition" for oxidability of a 3dimensional theory to $D>3$ dimensions is that the (very) long roots of $\bar{B}$ must be non degenerate, since the affine root, which is the magnetic wall $\beta^{1}-\theta\left(\phi^{\Delta}\right)$ in three dimensions, becomes a symmetry wall in $D>3$ dimensions (and the degeneracy of $\beta^{1}-\theta\left(\phi^{\Delta}\right.$ ) is the degeneracy of the (very) long highest root $\theta\left(\phi^{\Delta}\right)$ ). This necessary condition on the highest root was spelled out in [12]. It provides an obstruction to oxidation to $D>3$ dimensions for the following cases (see [21]) for conventions):

A II $\left(S U^{*}(2 n) / S p(n)\right)$ for which $m_{\theta}=4 ; B I I$ and $D I I(S O(p, 1))$ for which $m_{\theta}=p-1$; $C I I(S p(p, q) / S p(p) \times S p(q))$ for which $m_{\theta}=3 ; E I V\left(E_{6(-28)} / F_{4}\right)$ for which $m_{\theta}=8$; and $F I I\left(F_{4(-20)} / S O(9)\right)$ for which $m_{\theta}=7$. Here, $m_{\theta}$ is the multiplicity of the highest root $\theta$. That these non-split theories cannot be oxidized agrees with the findings of [23, 16].

It might help to notice that this list is precisely the list of real forms whose Satake diagrams have a "compact" Cartan generator at the simple root(s) that connects (or connect) to the affinizing root or equivalently that is not orthogonal to the most negative root of the simple Lie algebra under consideration. Affinization occurs in 2 dimensions; in the build-up of the $S L\left(D_{\max }-3, R\right) \times R$ chain one would expect the dilation of the fourth coordinate to be a noncompact Cartan generator at that vertex as soon as $D_{\max } \geq 4$ [14].

Conversely the fact that the root before the affinizing one is noncompact, to use approximate language, seems to imply that the affine root is noncompact too and can participate in a $G L\left(D_{\max }-2, R\right)$ group of duality symmetries [14].

Finally, we note that the $C I I$ (with $q=2$ ) and $F I I$ cases have a supersymmetric extension with an odd number of 3-dimensional supersymmetries $(N=5$ and $N=9$, respectively) [22]. These supersymmetries cannot come from four dimensions since a fourdimensional theory yields an even number of supersymmetries in $D=3$. Our obstruction to oxidation given above does not rely on supersymmetry, however, and prevents even non-supersymmetric "parents".

\section{Maximal dimensions}

When the theory can be oxidized, one can infer the maximal dimension(s) in which it can be formulated from the rules recalled above: this is $D_{\max }=k+2$ where $k$ is the length of the symmetry wall chain, i.e. the length of the single-linked chain of nodes in the

\footnotetext{
${ }^{3}$ For pure gravity, one curvature wall, namely $2 \beta^{1}+\beta^{2}+\cdots+\beta^{d-2}$ defines also a simple root. This case is covered in $[10]$.
} 
Dynkin diagram of the restricted root system $\bar{B}^{\wedge \wedge}$ that can be constructed starting from the overextended root, without loop (hereafter called "A-chain"). If there is a fork, each branch yields an independent maximal dimension. By mere inspection of the tables and Satake diagrams in [21] and of the Dynkin diagrams of the overextensions given e.g. in [11, 12], one easily gets the results collected in the following table:

\begin{tabular}{|l|l|c|}
\hline Class & Noncompact Symmetric Space $\mathrm{D}=3$ & $D_{\max }$ \\
\hline$A I$ & $S L(r+1) / S O(r+1)$ & $r+3$ \\
\hline$A I I I$ and $A I V$ & $S U(p, q) /\left(S\left(U_{p} \times U_{q}\right)\right)$ & 4 \\
\hline$B I$ and $D I$ & $S O(p, q) /(S O(p) \times S O(q)), p \geq q>1$ & $q+2$ and $6(q \geq 3)$ \\
\hline$C I$ & $S p(n, R) / U(n)$ & 4 \\
\hline$D I I I$ & $S O^{*}(2 n) / U(n)$ & 4 \\
\hline$E I$ & $E_{6(6)} / S p(4)$ & 6 \\
\hline$E I I$ & $E_{6(2)} /(S U(6) \times S U(2))$ & 4 \\
\hline$E I I I$ & $E_{6(-14)} /(S O(10) \times U(1))$ & 8 and 10 \\
\hline$E V$ & $E_{7(7)} / S U(8)$ & 4 \\
\hline$E V I$ & $E_{7(-5)} /(S O(12) \times S U(2))$ & 10 and 11 \\
\hline$E V I I$ & $E_{7(-25)} /\left(E_{6(-78)} \times U(1)\right)$ & 6 \\
\hline$E V I I I$ & $E_{8(8)} / S O(16)$ & 6 \\
\hline$E I X$ & $E_{8(-24)} /\left(E_{7(-133)} \times S U(2)\right)$ & 5 \\
\hline$F I$ & $F_{4(4)} /(S p(3) \times S U(2))$ & \\
\hline$G$ & $G_{2(2)} / S O(4)$ & \\
\hline
\end{tabular}

In all maximal oxidation dimensions, a theory which correctly reduces to $D=3$ actually exists. These theories are listed in [16] in terms of previously constructed models (23, 24, 25]).

It is interesting to observe that the same conclusions can be reached from other approaches to the hidden symmetries of gravitational theories. In the approach where the forms are associated with the roots of a superalgebra [18]-20], one needs only replace the couple of the affine and extended roots by the $D=3$ fermionic root, which must be of multiplicity one if the theory comes from a higher $(D>3)$ dimension; the rest of the discussion proceeds in the same way. A real chain $\mathfrak{s l}(n \mid 1)$ in the $D=3$ superalgebra (with long roots) allows to reach dimension $D=3+n$. Reduction on an $n$-torus gives indeed $n$ odd roots $E_{i}$ $(i=1, \ldots, n)$ which combine into a $\mathfrak{s l}(n \mid 1)$ algebra, with simple roots $E_{0}-E_{1}, \ldots, E_{n-1}-E_{n}$ (even) and $E_{n}$ (odd). When considering the restricted roots system, as we do in the billiard approach, it corresponds to a nondegenerate $\mathfrak{s l}(n \mid 1)$ chain.

In the approach where it is the triple extension that appears [26, 27], one gets the same conclusion because the overextended part is identical.

\section{5. $p$-form spectrum and oxidation}

The billiard provides also information on the $p$-forms that must be present in the maximal oxidation dimension because one knows how the $p$-form walls must connect to the A-chain. 
A simple electric $p$-form wall connects to the $p$-th root in the A-chain (counting now from the root at the end of the A-chain opposite to the overextended root). A simple magnetic $p$-form wall connects to the $(d-p-1)$-th root. One also knows the multiplicities. Together with the Weyl group, this can be used to construct the $p$-form spectrum and determine the dilaton couplings.

Rather than deriving explicitly all cases, we shall focus on $E I X$, i.e., $E_{8(-24)} /\left(E_{7(-133)} \times\right.$ $S U(2))$, which has a restricted root system of $F_{4}$-type

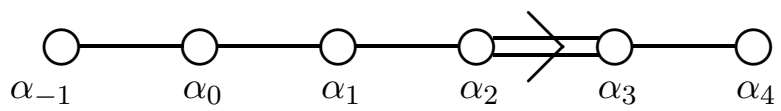

The other cases are treated similarly and reproduce known results. The root $\alpha_{-1}$ in the above Dynkin diagram is the overextended root, the root $\alpha_{0}$ is the affine root, while $\alpha_{1}$ and $\alpha_{2}$ are the long roots of $F_{4}$. The roots $\alpha_{-1}, \alpha_{0}, \alpha_{1}, \alpha_{2}$ have multiplicity 1 . The roots $\alpha_{3}$ and $\alpha_{4}$ are the short roots and have multiplicity 8 [21]. The A-chain is given by the roots $\alpha_{-1}, \alpha_{0}, \alpha_{1}, \alpha_{2}$, which read, in $D_{\max }=6$ dimensions, $\alpha_{2}=\beta^{2}-\beta^{1}, \alpha_{1}=\beta^{3}-\beta^{2}$, $\alpha_{0}=\beta^{4}-\beta^{3}$ and $\alpha_{-1}=\beta^{5}-\beta^{4}$. Since $F_{4}^{\wedge \wedge}$ has rank 6 and since there is only five logarithmic scale factors, one needs one dilaton.

Because the short root $\alpha_{3}$ is attached to the first root $\alpha_{2}$ of the A-chain, it corresponds to the electric wall of a 1-form. Requiring that the root has length squared equal to one (one has $\left(\alpha_{2} \mid \alpha_{2}\right)=2$ and $\left.\left(\alpha_{2} \mid \alpha_{3}\right)=-1\right)$ fixes the dilaton coupling of the one-form to $\lambda^{(1+)}=-1$, i.e., $\alpha_{3}=\beta^{1}-(\phi / 2)$. The last simple root $\alpha_{4}$ is not attached to the A-chain: it corresponds therefore to an axion, with dilaton coupling equal to $\lambda^{(0)}=2$, i.e., electric 0 -form wall $\alpha_{4}=\phi$. The degeneracy of the short roots is 8 ; hence, at this stage, we need eight 1 -forms and eight 0 -forms.

This is not the entire spectrum of $p$-forms because we have only accounted so far for the simple roots. There are other positive roots in the theory which correspond also to walls of the $p$-form type, as can be seen by acting with the finite Weyl group of $F_{4}$ (= restricted Weyl group of $\mathcal{U}_{3}$ ) on the roots $\alpha_{1}, \alpha_{2}, \alpha_{3}$ and $\alpha_{4}$. These roots must be included by incorporating the corresponding $p$-forms.

By Weyl-reflecting the short root $\alpha_{3}=\beta^{1}-(\phi / 2)$ in $\alpha_{4}$, we get the root $\beta^{1}+(\phi / 2)$. This is also an electric 1-form wall, with degeneracy 8 . We thus need eight further 1-forms, with dilaton couplings $\lambda^{(1-)}=1$. We then observe that the symmetry wall reflection in $\beta^{2}-\beta^{1}$ replaces $\beta^{1}-(\phi / 2)$ by $\beta^{2}-(\phi / 2)$. Reflecting this root in $\beta^{1}+(\phi / 2)$ yields $\beta^{1}+\beta^{2}$, which is the electric (= magnetic) wall of a chiral 2 -form with zero dilaton coupling. This root is short, so again degenerate 8 times: we need eight such chiral 2-forms (or four non-chiral 2-forms with zero dilaton couplings). Finally, by reflecting in $\alpha_{3}$ the long root $\beta^{2}-\beta^{1}$, we generate the long root $\beta^{1}+\beta^{2}-\phi$. This is an electric wall for a 2 -form with dilaton coupling $\lambda^{(2)}=-2$, which we must include. A reflection in $\alpha_{4}$ yields $\beta^{1}+\beta^{2}+\phi$ but this is just a magnetic root for the same 2 -form, so we do not need any further additional field to get these walls in the Lagrangian. Similarly, the short roots $\beta^{1}+\beta^{2}+\beta^{3} \pm(\phi / 2)$ obtained by reflecting $\beta^{3} \pm(\phi / 2)$ in $\beta^{1}+\beta^{2}$ are the magnetic walls of the 1 -forms and do not need new fields either. [Note in passing the obvious misprints in formulas (6.21) and 
(6.22) for the magnetic walls of the 2 -forms in [11]. (Also the magnetic walls of $G_{2}$ are miswritten there).]

To summarize: by acting with the finite Weyl group of $F_{4}$ on the simple roots $\alpha_{1}, \alpha_{2}$, $\alpha_{3}$ and $\alpha_{4}$, we get all the roots of $F_{4}$. The positive roots, which must have a term in the Lagrangian, are $\beta^{i}-\beta^{j}(i>j)$ (long), $\phi$ (short), $\beta^{i} \pm(\phi / 2)$ (short), $\beta^{i}+\beta^{j}(i<j)$ (short), $\beta^{i}+\beta^{j} \pm \phi(i<j)$ (long), $\beta^{i}+\beta^{j}+\beta^{k} \pm(\phi / 2)(i<j<k)$ (short) and $2 \beta^{i}+\beta^{j}+\beta^{k}(i \neq j$, $i \neq k, j<k$ ) (long). Here, $i, j, k \in\{1,2,3\}$. These are all the 24 positive roots of $F_{4}$. They are all accounted for by the $p$-form walls, except the last ones, which are curvature walls following from the Einstein-Hilbert action (and the symmetry walls). By acting with the $F_{4}^{\wedge}$-Weyl reflections associated with the other symmetry walls, one covariantizes the above expressions (i.e., one generates the same walls but with $i, j, k \in\{1,2,3,4,5\}$ ). Continuing, i.e., acting with the other elements of the infinite Weyl group of $F_{4}^{\wedge \wedge}$, one generates new walls but these are not of the $p$-form type because they necessarily contain a $\beta^{i}$ with a coefficient at least 2 (except the magnetic walls $\beta^{i}+\beta^{j}+\beta^{k}+\beta^{m}-\phi(i<j<k<m$ ) of the 0 -form and the null magnetic walls $\beta^{i}+\beta^{j}+\beta^{k}+\beta^{m}$ of the dilaton, but these are already accounted for). All the $p$-form roots have been exhausted.

The superalgebra approach gives an alternative procedure to generate the $p$-spectrum from the Borcherds-Chevalley-Serre relations and also provides detailed information on the Chern-Simons terms in the Lagrangian (as the group theory approach [15]-117] and the principles given in 27] do). See [18, 20] for the split case and [19, 20] for the non split case. In this formalism, there are roots associated to scalars but also to nonreduced $p$-forms. If $\alpha$ is a root associated to a $p$-form in the superalgebra of the $D$-dimensional theory, one gets in addition in lower dimensions roots like $\alpha-E_{i}$ (corresponding to a $(p-1)$-form), $\alpha-E_{i}-E_{j}((p-2)$-form $) \ldots$ All original roots remain in the spectrum, and the oxidation procedure computes precisely those roots which are present in the oxidised theory.

If we consider again the $E I X$ example, the superalgebra of the $D=3$ theory is given by the following Satake diagram:

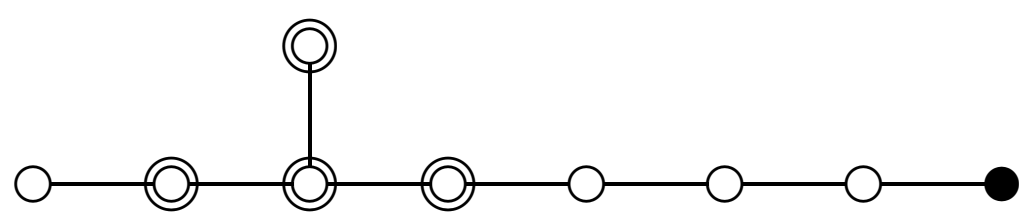

where white dots are even simple roots of degree 0 and the black one an odd $\mathfrak{s l}(1 \mid 1)$ one of degree 1; the circled roots are those which are antiinvariant under the Cartan involution defining the real form. One sees on this diagram an $\mathfrak{s l}(3 \mid 1)$ chain that is invariant under the Cartan involution (its 3 simple roots are the rightmost ones which are orthogonal to, ie disconnected from, the antiinvariant ones). It means that we can oxidise the model up to $D=3+3=6$.

The superalgebra of the maximal oxidised theory can be easily deduced; it is given by the following diagram: 


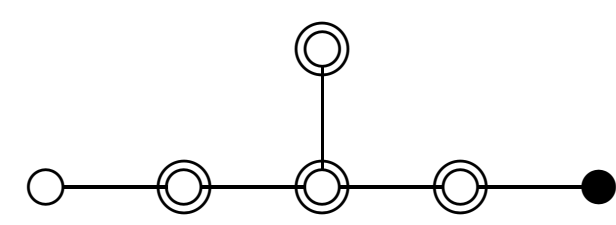

We see that the real rank of this superalgebra is 2 , from what we know there is $2-1=1$ dilaton in the spectrum. We can also compute that there are 10 degree zero positive roots, so 8 -forms; there are also 16 degree 1 roots, obtained by acting on the odd simple root with the Weyl group of $D_{5}$, which gives us 161 -forms. There are 10 degree 2 roots, but related by duality: there are only 5 dynamical 2 -forms ( 2 chiral 2 -forms count as a single ordinary 2-form). In addition we find all magnetic duals of the dilaton, 0 - and 1-forms: 16 3 -forms and 8+1 4-forms.

\section{Results and comments}

We have shown in this paper how the billiard analysis of gravitational theories, related to Satake diagrams and restricted root systems [12], provides useful information on their oxidation endpoint both in the split and in the non-split cases. Note, in particular, that our results suggest a 6-dimensional formulation of magic square supergravity theories [28, 29] since these have a $F_{4}$ restricted root system, this was alrealdy announced a few years ago [30]. The real form $E I X$ with the $D=6$ spectrum displayed above would in fact correspond to the octonion case.

It is noteworthy that the split real forms allow oxidation by at least one dimension, to $D \geq 4$. One is stuck to $D=3$ only for certain non-split real forms, because of the non trivial multiplicities of the (very) long roots (which are always non-degenerate in the split case). The constraint on multiplicities provides a rather powerful insight. Finally, we mention the recent paper [31] which also deals with $\mathcal{U}_{3}$, billiards and oxidation.

\section{Acknowledgments}

Marc Henneaux is grateful to the organizers of the 27th Johns Hopkins workshop and of the 36th International Symposium Ahrenshoop where he presented the results of the papers [5, 9, 6, 11, 12]. The work of S.d.B., M.H. and L.P. is supported in part by the "Actions

de Recherche Concertées" of the "Direction de la Recherche Scientifique - Communauté Française de Belgique", by a "Pôle d'Attraction Interuniversitaire" (Belgium) and by IISNBelgium (convention 4.4505.86). Support from the European Commission RTN programme HPRN-CT-00131, in which S.d.B., M.H. and L.P. are associated to K. U. Leuven, is also acknowledged.

\section{References}

[1] V.A. Belinskii, I.M. Khalatnikov and E.M. Lifshitz, Adv. Phys. 19, 525 (1970).

[2] V.A. Belinskii, I.M. Khalatnikov and E.M. Lifshitz, Adv. Phys. 31, 639 (1982). 
[3] D.M. Chitre, Ph. D. thesis, University of Maryland, 1972.

[4] C.W. Misner, in: D. Hobill et al. (Eds), Deterministic chaos in general relativity, Plenum, 1994, pp. 317-328 [gr-qc/9405068].

[5] T. Damour and M. Henneaux, Phys. Rev. Lett. 85, 920 (2000) [arXiv:hep-th/0003139].

[6] T. Damour, M. Henneaux and H. Nicolai, Class. Quant. Grav. 20, R145 (2003) [arXiv:hep-th/0212256].

[7] B. Julia, in Lectures in Applied Mathematics, AMS-SIAM, vol 21 (1985), p.355.

[8] P. Goddard and D. I. Olive, "Algebras, Lattices And Strings," DAMTP-83/22 Talks given at the Workshop on Vertex Operators in Mathematics and Physics, Berkeley, Calif..

[9] T. Damour and M. Henneaux, Phys. Rev. Lett. 86, 4749 (2001) [arXiv:hep-th/0012172].

[10] T. Damour, M. Henneaux, B. Julia and H. Nicolai, Phys. Lett. B 509, 323 (2001) [arXiv:hep-th/0103094].

[11] T. Damour, S. de Buyl, M. Henneaux and C. Schomblond, JHEP 0208, 030 (2002) [arXiv:hep-th/0206125].

[12] M. Henneaux and B. Julia, JHEP 0305, 047 (2003) [arXiv:hep-th/0304233].

[13] B. Julia, in Superspace and supergravity Talk given M at the Nuffield Workshop Cambride UK, 1980 ed. M. Rocek and S. Hawking.M

[14] B. Julia, in Strings, branes and dualities Lectures at M Carg ese School 1997, Kluwer (1999). They contain a review of the "Silver M rules of group disintegrations". They also contain a general conjecture $\mathrm{M}$ on the triple extensions like $E^{\wedge \wedge \wedge} \mathrm{M}$ (paper in preparation). $\mathrm{M}$

[15] A. Keurentjes, Nucl. Phys. B 658, 303 (2003) [arXiv:hep-th/0210178].

[16] A. Keurentjes, Nucl. Phys. B 658, 348 (2003) [arXiv:hep-th/0212024].

[17] A. Keurentjes, Class. Quant. Grav. 20, S525 (2003) [arXiv:hep-th/0212050].

[18] P. Henry-Labordere, B. Julia and L. Paulot, JHEP 0204, 049 (2002) [arXiv:hep-th/0203070].

[19] P. Henry-Labordere, B. Julia and L. Paulot, JHEP 0304, 060 (2003) [arXiv:hep-th/0212346].

[20] P. Henry-Labordere, B. Julia and L. Paulot, in preparation

[21] S. Helgason, Differential Geometry, Lie Groups and Symmetric Spaces, Graduate Studies in Mathematics vol.34, AMS 2001 printing.

[22] B. de Wit, A. K. Tollsten and H. Nicolai, Nucl. Phys. B 392, 3 (1993) [arXiv:hep-th/9208074].

[23] P. Breitenlohner, D. Maison and G. W. Gibbons, Commun. Math. Phys. 120, 295 (1988).

[24] E. Cremmer, B. Julia, H. Lu and C. N. Pope, arXiv:hep-th/9909099.

[25] A. Sagnotti, Phys. Lett. B 294, 196 (1992) [arXiv:hep-th/9210127];

B. de Wit and A. Van Proeyen, Commun. Math. Phys. 149, 307 (1992) [arXiv:hep-th/9112027];

R. D'Auria, S. Ferrara and C. Kounnas, Phys. Lett. B 420, 289 (1998) [arXiv:hep-th/9711048];

B. de Wit, F. Vanderseypen and A. Van Proeyen, Nucl. Phys. B 400, 463 (1993) [arXiv:hep-th/9210068]. 
[26] F. Englert, L. Houart, A. Taormina and P. West, JHEP 0309, 020 (2003) [arXiv:hep-th/0304206].

[27] F. Englert, L. Houart and P. West, JHEP 0308, 025 (2003) [arXiv:hep-th/0307024].

[28] M. Gunaydin, G. Sierra and P. K. Townsend, "The Geometry Of N=2 Maxwell-Einstein Supergravity And Jordan Algebras," Nucl. Phys. B 242, 244 (1984).

[29] M. Gunaydin, G. Sierra and P. K. Townsend, "Exceptional Supergravity Theories And The Magic Square," Phys. Lett. B 133, 72 (1983).

[30] B. Julia, talk Sept 7, 1999 at TMR meeting on Quantum aspects of gauge theories, supersymmetry and unification Paris.

[31] P. Fre, V. Gili, F. Gargiulo, A. Sorin, K. Rulik and M. Trigiante, arXiv:hep-th/0309237. 